\title{
Collins and Sivers Asymmetries from COMPASS
}

\author{
Andrea Bressan* \\ (on behalf of the COMPASS collaboration) \\ CERN, 1217 Geneve 23, Switzerland \\ E-mail: Andrea.Bressan@cern.ch
}

\begin{abstract}
In the years 2002-2004 the COMPASS experiment has collected data with a polarized ${ }^{6} \mathrm{LiD}$ target and a $160 \mathrm{GeV}$ muon beam. About $20 \%$ of the running time has been devoted to measure transverse spin effects in semi inclusive deep inelastic scattering, one of the main objectives of the COMPASS spin program. The results for the Collins and the Sivers asymmetries, both for unidentified and identified hadrons, are presented here. The measured asymmetries on the ${ }^{6} \mathrm{LiD}$ target are small and compatible with zero within the few percent statistical errors, an important result which can be interpreted as cancellation between $\mathrm{u}$ and $\mathrm{d}$ quark contribution in the deuteron and which allows to better constrain the parton distribution functions.
\end{abstract}

\section{The Collins and Sivers mechanism}

At twist-two level, three independent quark distribution functions are needed to describe the quark spin content of the nucleon; the momentum distribution $q(x)$, the helicity distribution $\Delta q(x)$ and the transversity distribution $\Delta_{T} q(x)$. At variance with $q$ and $\Delta q, \Delta_{T} q$ is chiralodd and cannot be measured in inclusive deep inelastic scattering (DIS), which is the main source of information on the nucleon partonic structure [2]. However, it can be measured in semi-inclusive DIS (SIDIS) in combination with chiral-odd fragmentation functions, such as the the Collins fragmentation function $\Delta D_{a}^{h}$ for hadron production, and the interference fragmentation function $H_{1}^{\varangle}$ for hadron pair production [3], giving rise to an azimuthal single spin asymmetry (SSA) in the final state hadrons [4].

The Collins mechanism is not the only way to create SSA for single hadrons distributions; a different mechanism, where SSA arises from correlation between the nucleon spin and the quark intrinsic transverse momentum $k_{T}$ was proposed by Sivers [5] and is described by the so called Sivers distribution function, $\Delta_{0}^{T} q\left(x, k_{T}\right)$. The Collins and Sivers effects can be easily disentangled in SIDIS since they are related to asymmetries in two independent azimuthal angles. The general expression for the single hadron production cross sections contains six more transverse target polarization dependent azimuthal asymmetries, which also have been extracted in COMPASS; for a review of the results see [6].

The Collins mechanism leads to a modulation in the azimuthal distribution of the inclusively produced hadrons given by:

$$
N\left(\Phi_{C}\right)=\alpha\left(\Phi_{C}\right) \cdot N_{0}\left(1+A_{\mathrm{Col}} \cdot P_{T} \cdot f \cdot D_{N N} \sin \Phi_{C}\right),
$$

where the function $\alpha$ contains the apparatus efficiency and acceptance, $P_{T}$ is the target polarization $(\sim 50 \%), D_{N N}=(1-y) /\left(1-y+y^{2} / 2\right)$ is the spin transfer coefficient and $f$ is the target dilution factor, i. e. the fraction of polarizable nuclei in the target $(\sim 40 \%)$; $\Phi_{C}=\phi_{h}-\phi_{S^{\prime}}=\phi_{h}+\phi_{S}-\pi$ is the Collins angle, with $\phi_{h}$ the hadron azimuthal angle and

*on leave of absence from Università degli Studi di Trieste and INFN - Sezione di Trieste 
$\phi_{S^{\prime}}$ the final azimuthal angle of the quark spin and $\phi_{S}$ the azimuthal angle of the nucleon spin in the $\gamma-N$ system. Finally

$$
A_{\mathrm{Col}}=\frac{\sum_{q} e_{q}^{2} \cdot \Delta_{T} q(x) \cdot \Delta D_{q}^{0}\left(z, p_{T}^{h}\right)}{\sum_{q} e_{q}^{2} \cdot q(x) \cdot D_{q}^{h}\left(z, p_{T}^{h}\right)}
$$

is the Collins asymmetry, arising from the product of the transversity distribution $\Delta_{T} q$ and the Collins fragmentation function $\Delta D_{q}^{0}$ (the quantities in the denominator are the known momentum distribution $q$ and the unpolarized fragmentation function $D_{q}^{h}$ ).

The azimuthal modulation arising from the Sivers mechanism can be written as:

$$
N\left(\Phi_{S}\right)=\alpha\left(\Phi_{S}\right) \cdot N_{0}\left(1+A_{\mathrm{Siv}} \cdot P_{T} \cdot f \sin \Phi_{S}\right),
$$

with a modulation expressed in terms of the Sivers angle $\Phi_{S}=\phi_{h}-\phi_{S}$, where again $\phi_{h}$ the hadron azimuthal angle and $\phi_{S}$ the azimuthal angle of the nucleon spin. The Siver asymmetry is defined as:

$$
A_{\mathrm{Siv}}=\frac{\sum_{q} e_{q}^{2} \cdot \Delta_{0}^{T} q\left(x, p_{T}^{h}\right) \cdot D_{q}^{h}(z)}{\sum_{q} e_{q}^{2} \cdot q(x) \cdot D_{q}^{h}(z)}
$$

Since in this case the unpolarized fragmentation functions are known, the measurement of the Siver asymmetry for both positive and negative produced hadrons allows directly to extract the Siver functions, if the measured asymmetry are different from zero, while a zero result for an isoscalar target like the one used in COMPASS can come both from a vanishing Sivers function or from a cancellation between $u$ and $d$ quark contributions.

\section{Results}

The event selection requires standard DIS cuts, i.e. $Q^{2}>1((\mathrm{GeV}) / \mathrm{c})^{2}$, mass of the final hadronic state $W>5 \mathrm{GeV} / c^{2}, 0.1<y<0.9$, and the detection of at least one hadron in the final state. For the detected hadrons it is also required that:

- the fraction of the virtual photon energy carried is $z\left(=E_{h} / E_{\gamma}\right)>0.2$ to select hadron from the current fragmentation region (for the leading hadrons, i.e. the hadron with the largest $z$ in the event, the cuts selection is more strict: $z>0.25$ and, in case that the missing $z_{\text {miss }}=1-\sum_{h} z_{h}>z_{\text {leading, }}$, no neutral blob in the calorimeters with an energy larger than that of the leading hadron);

- $p_{T}>0.1 \mathrm{GeV} / c$ (where $p_{T}$ is the hadron transverse momentum with respect to the virtual photon direction) for a better determination of the azimuthal angle $\phi_{h}$.

The resulting Collins and Sivers asymmetries from the whole deuteron data for all hadrons of the event fulfilling the described criteria are shown in figure 1 [7], with the error bars accounting only for the statistical uncertainty. The asymmetries are measured as a function of $x_{\mathrm{Bj}}, z$ and $p_{T}^{h}$. Different sources of systematic effects have been checked, showing that systematic errors are much smaller than the statistical uncertainty. Both the Collins and Sivers asymmetries turned out to be small and compatible with zero; the same behavior is observed when only leading hadrons are considered [7].

Even if there were theoretical predictions for small asymmetries (given an opposite contribution for the $u$ and $d$ quark, resulting in a large cancellation for isoscalar targets like the 


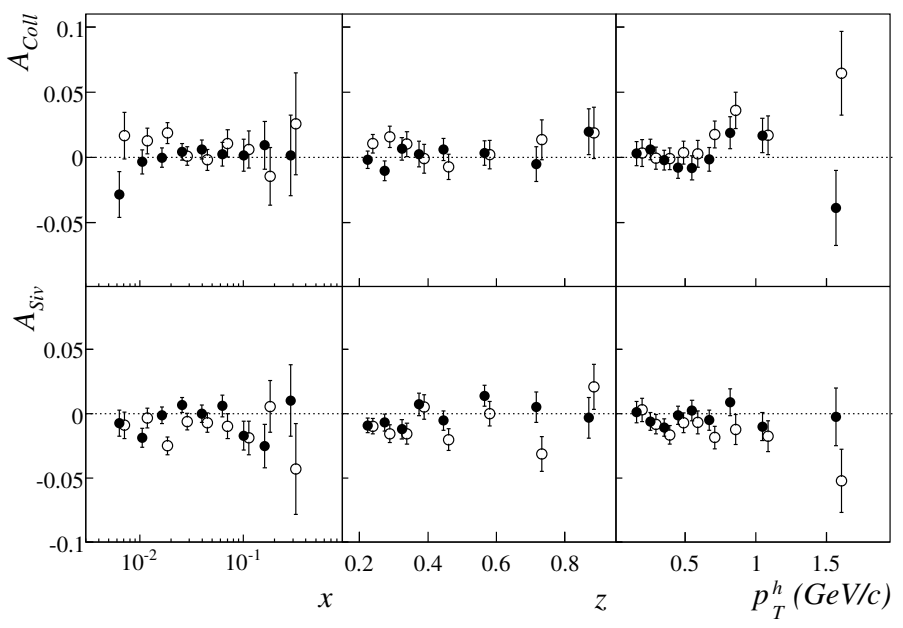

Figure 1: Collins and Sivers asymmetry for positive (full points) and negative (open points) hadrons as a function of $x_{\mathrm{Bj}}, z$ and $p_{T}^{h}$.

deuteron), it was not obvious that the measured asymmetries have been so small. For what concerns the Collins mechanism, HERMES have measured (on the $u$-dominated proton) a non-zero Collins asymmetry, providing a convincing evidence that both the transversity distribution $\Delta_{T} u(x)$ and the Collins fragmentation function $\Delta_{T}^{0} D_{u}^{h}(z)$ are different from zero. An independent evidence that the Collins mechanism is a sizable effect came by the recent analysis of the Belle collaboration, which is measuring the azimuthal distribution in the $e^{+} e^{-} \rightarrow h^{+} h^{-} X$ reaction [8].

Recently, a global analysis of the HERMES, COMPASS and Belle data has allowed a first extraction of the transverity distributions [9], while for a global analysis of COMPASS and HERMES results for the Sivers asymmetry see [10].

The sizable Sivers asymmetry measured by the HERMES collaboration in the case of positive pions produced on protons, is indicating that the Sivers mechanism is also at work. The zero result of COMPASS for the Sivers asymmetries of both positive and negative hadrons suggest that $\Delta_{0}^{T} d \sim-\Delta_{0}^{T} u$.

The same analysis done for the charged hadrons has been repeated to extract the Collins and Sivers asymmetries for $\pi^{ \pm}$and $\mathrm{K}^{ \pm}$, using the information of the RICH detector to identify the hadrons. The COMPASS RICH1 detector uses $\mathrm{C}_{4} \mathrm{~F}_{10}$ as radiator gas, with a refractive index of $\sim 1.0015$ for $7 \mathrm{eV}$ Cerenkov photons, that gives a 2 (9) GeV threshold for pions (kaons), and allows to separate pions from kaons up to $45-50 \mathrm{GeV}$. More than $80 \%$ of the hadrons in this energy interval identified by the RICH are pions and the rest are kaons. The measured asymmetries for pions are similar to those shown in fig. 1; the Collins and Sivers asymmetries for kaons are shown in figure 2. Also in this case the measured asymmetries are small, and compatible with zero; the only indication for a signal, both positive and negative kaons, is visible in the Collins asymmetries as a function of $x$ in the $x \sim 0.1$ region. Since it is only one point at two-three standard deviations from zero, this observation needs to be confirmed by more precise measurements. 


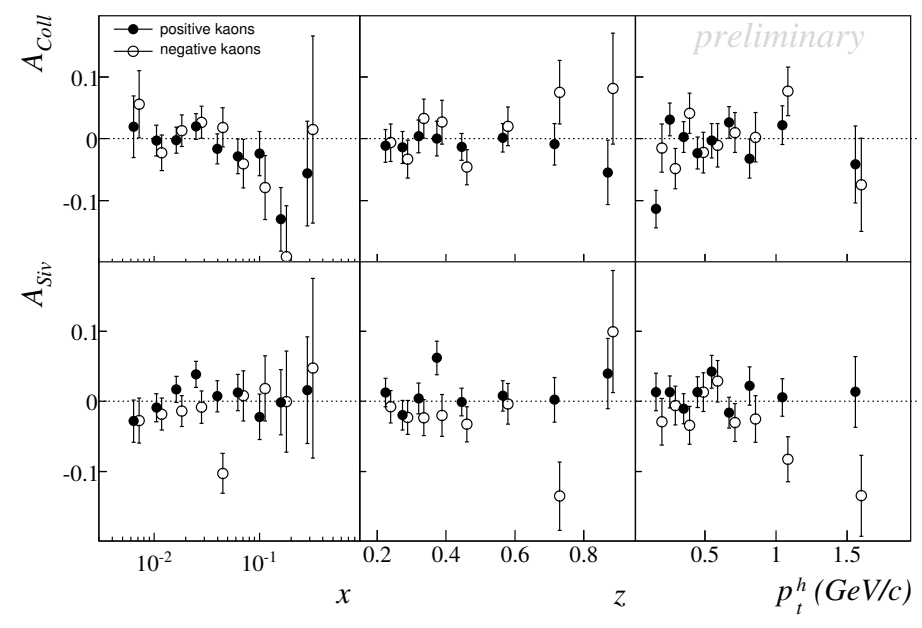

Figure 2: Collins and Sivers asymmetry for positive (full points) and negative (open points) kaons as a function of $x_{\mathrm{Bj}}, z$ and $p_{T}^{h}$.

The COMPASS 2007 run will be devoted to the data taking with a polarized proton $\left(\mathrm{NH}_{3}\right)$ target, and the data will allow to measure the Collins and Sivers asymmetries at the same energy of the deuteron results presented here.

\section{References}

[1] Slides:

http://indico. cern. ch/contributionDisplay . py? contribId=151\&sessionId=4\&conf Id=9499

[2] see, f.i., V. Barone, A. Drago and P. G. Ratcliffe, Phys. Rept. 359 (2002) 1.

[3] C. Schill, "Transversity signals in two-hadron production at COMPASS", these proceedings.

[4] J. C. Collins, Nucl. Phys. B 396 (1993) 161.

[5] D. W. Sivers, Phys. Rev. D 41 (1990) 83.

[6] A. Kotzinian, "Beyond Collins and Sivers: further measurements of target transverse spin-dependent azimuthal asymmetries in semi-inclusive DIS from COMPASS", these proceedings.

[7] The COMPASS Collaboration, E.S. Ageev et. al, Nucl. Phys. B 765 (2007) 31

[8] The Belle Collaboration, K. Abe et al., Phys. Rev. Lett. 96 (2006) 232002.

[9] M. Anselmino et al., Phys. Rev. D 75, 054032 (2007) and [arXiv:hep-ph/0701006v1].

[10] M. Anselmino et al., Phys. Rev. D 72, 094007 (2005) 